\title{
Overview on the connection between reactive Kripke models and argumentation networks
}

\author{
Dov M. Gabbay
}

Published online: 22 September 2012

(C) Springer Science+Business Media B.V. 2012

The collection in this special issue contains mainly papers on reactive Kripke semantics and on argumentation. This overview says a few words about how these papers fit in the general picture.

\section{Reactive arrows and modalities}

Modal logic $\mathbf{K}$ is complete for tree Kripke frames. These have the form $(S, R, 0)$, where $S$ is a non-empty set of worlds and $R \subseteq S^{2}$ is a tree relation on $S$ with root point 0 . Being a tree means that for every point $x \in S$, there exists a unique path $\left(0, x_{1}, \ldots, x_{n}\right)$ leading from 0 to $x_{n}=x$, such that $0 R x_{1}, x_{1} R x_{2}, \ldots, x_{n-1} R x_{n}$ hold. The option $n=1$ means $0 R x$.

We add reactivity to such a model by adding a further set of relations $R^{\prime} \subseteq S^{2} \times S^{2}$.

We write $x \rightarrow y$ when $(x, y) \in R$ and we write $(x \rightarrow y) \rightarrow(u \rightarrow v)$ when $((x, y),(u, v)) \in R^{\prime}$. The first are called arrows and the second are called double arrows.

Figure 1 is an example of a reactive frame $\left(S, R, R^{\prime}, 0\right)$. Since $(S, R, 0)$ is a tree, Fig. 1 can be represented as Fig. 2.

How do we interpret the double arrows? We imagine an agent traversing the tree from point 0 along the arrows. This agent may be moving along the arrows in the course of evaluation of a modal formula. Let us take, for example, the formula $\diamond \diamond \diamond \square \perp$, and evaluate it at node 0 .

D. M. Gabbay

Bar-Ilan University, Ramat-Gan, Israel

D. M. Gabbay ( $\varangle)$

King's College London, Strand, London

e-mail: dov.gabbay@kcl.ac.uk

D. M. Gabbay

University of Luxembourg, Luxembourg, Germany 
Fig. 1 An example of a reactive frame

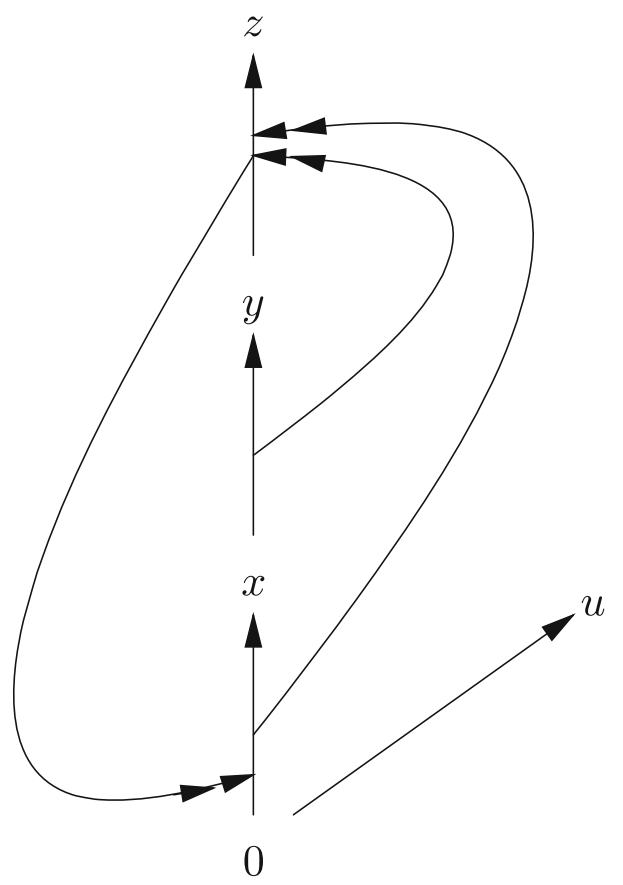

We need to find a path $\left(0, x_{1}, x_{2}, x_{3}\right)$ such that $x_{3} \vDash \square \perp$ holds and $0 R x_{1}$, $x_{1} R x_{2}, x_{2} R x_{3}$ hold. Such a path exists; it is $(0, x, y, z)$. Let the operators $\diamond$ and $\square$ look only at the relation $R$. $\diamond$ and $\square$ move along the arrows. They disregard the double arrows.

Fig. 2 Representing Fig. 1

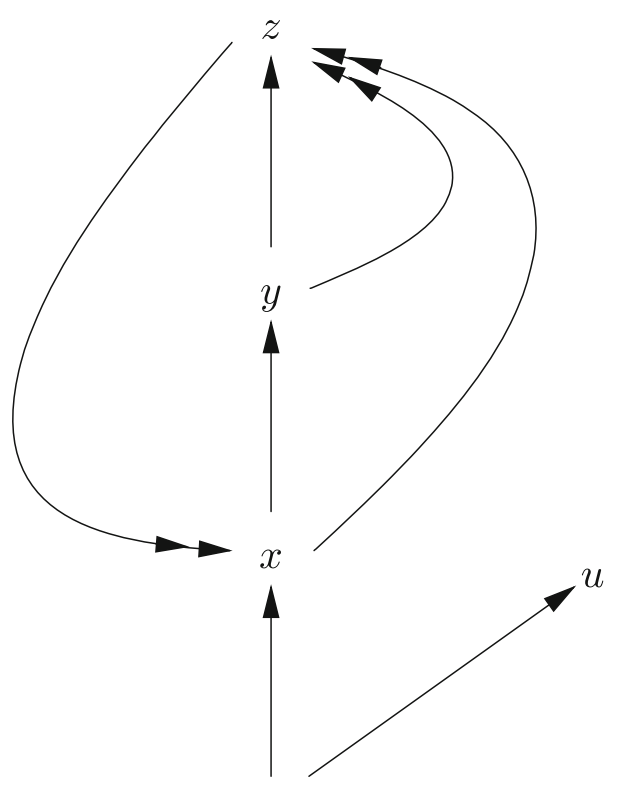


We now have to say what the double arrows do. We consider two options.

Option 1. Ordinary reactivity When we evaluate at a node $x_{m}$ such that $\left(0, x_{1}, \ldots, x_{m}\right)$ is the path leading to $x_{m}$, then we disconnect any arrow of the form $u \rightarrow v$ such that $\left(x_{i} \rightarrow x_{i+1}\right) \rightarrow(u \rightarrow v)$ holds for some $0 \leq i<m$, (i.e., we take $u \rightarrow v$ out of $R$ when the above double arrow is in $\left.R^{\prime}\right)$. So, for example, when we move along the path $(0, x, y)$ the arrow $y \rightarrow z$ gets disconnected because it is being hit twice; once from $(0 \rightarrow x) \rightarrow(y \rightarrow z)$ and once from $(x \rightarrow y) \rightarrow(y \rightarrow z)$. Let $\vee^{\prime}$ be a modality which respects the effects of the double arrows. Then we have

$$
\begin{aligned}
& 0 \vDash \Delta \diamond \square^{\prime} \perp \\
& 0 \not \forall \diamond \diamond \square \perp
\end{aligned}
$$

Note that the double arrow $(y \rightarrow z) \rightarrow(0 \rightarrow x)$ has no effect on the evaluation because it is triggered after we pass through the arrow $0 \rightarrow x$. It will have an effect if we have some operators which go backwards.

Option 2. Switch reactivity Switch reactivity counts the number of hits by double arrows which impact on an arc. If it is even, the arc remains connected. If it is odd then the arc is disconnected. Thus a switch double arrow switches a connection "on" if it is "off" and "off" if it is "on". So it acts like a switch. The default condition for arcs is that they are "on".

Thus in Fig. 1 the arc $y \rightarrow z$ shall remain on because it is hit twice by double arrows. So for the case of switch double arrows, $0 \not \forall \diamond \diamond \square^{\prime} \perp$.

Note that the reacctivity idea does not require $(S, R, 0)$ to be a tree. We can add reactivity and move to $\left(S, R, R^{\prime}, 0\right)$ for any Kripke frame. The tree property is used only in reducing $R^{\prime} \subseteq S^{2} \times S^{2}$ to an $R_{1} \subseteq S \times S$, since in a tree, the arc $x \rightarrow y$ is uniquely determined by the node $y$. In fact, we can allow for reflexivity at the root 0 as well if we understand $0 \rightarrow 0$ as $(0 \rightarrow 0) \rightarrow(0 \rightarrow 0)$. We can have this understanding only for $x=0$. If $x \neq 0$ then $y \rightarrow x$ exists for $y=\operatorname{predecessor}(x)$ and $x \rightarrow x$ must mean $(y \rightarrow x) \rightarrow(y \rightarrow x)$.

Of course if there is no double arrow $(y \rightarrow x) \rightarrow(y \rightarrow x)$ then $x \rightarrow x$ can mean $(x \rightarrow x) \rightarrow(x \rightarrow x)$. So in principle we can reduce arc reactivity $R^{\prime}$ to point to point relation $R_{1}$ in many more cases if we have the right conventions.

Fig. 3 Reactive frame not equivalent to any Kripke frame

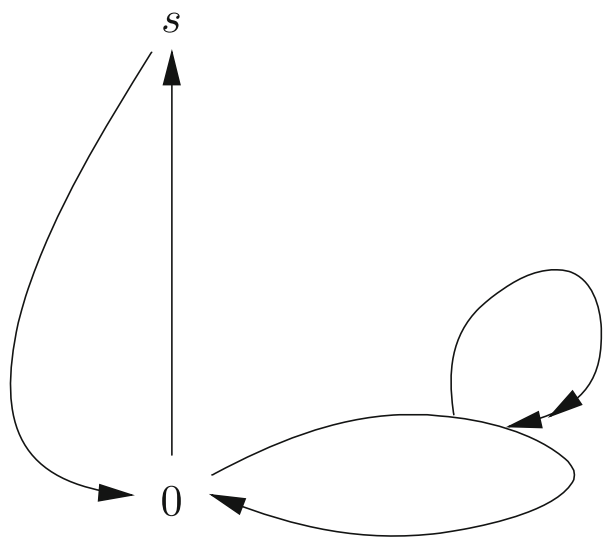


Fig. 4 Another representation of Fig. 3

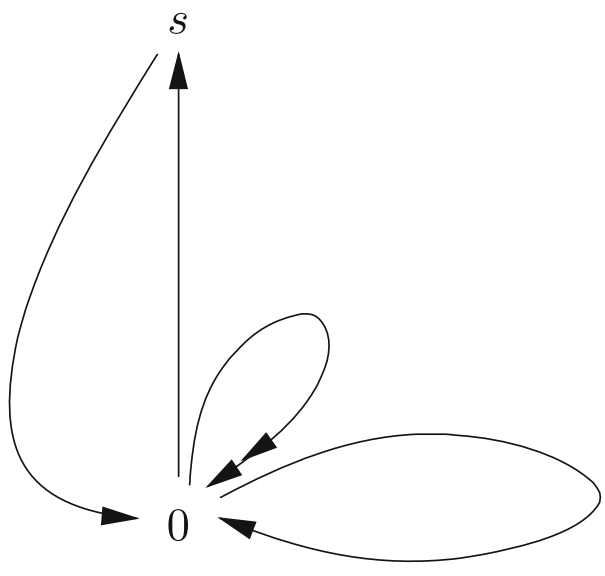

Consider Figs. 3 and 4.

The above figure gives rise to a modal logic with a single modaltiy $\square^{\prime}$ which is not complete for any ordinary class Kripke frames but is complete for the above reactive frame.

The above discussion places reactive Kripke structures somewhere in the area of bimodal logics. It yields uni-modal logical systems with some sort of a modality $\vee^{\prime}$ which is more expressive than a single traditional modality and less expressive than a bi-modal logical system with an independent additional modality.

This gives us scope for further research. We can consider the following questions:

1. Expressive power of $\diamond, \diamond^{\prime}$

2. Applications of the idea of reactivity

3. Axiomatisation, proof theory and completeness proofs for these modalities.

\section{Reactivity and argumentation}

Let us take another look at Fig. 2. This can be viewed as a bipolar argumentation frame. The nodes are arguments. Arguments can either attack or support other arguments. If argument $x$ supports argument $y$, we write $x \rightarrow y$. If argument $x$ attacks argument $y$ we write $x \rightarrow y$. Let $\rightarrow_{*}$ be the reflexive and transiitive closure of $\rightarrow$. The way we play the game in argumentation theory is, for example, to seek maximal subsets $\mathbb{E}$ of arguments defined as follows.

1. Let $x \rightarrow_{*} y$ be defined as meaning that for some $x^{\prime}, y^{\prime}$ we have that $y \rightarrow_{*} y^{\prime}$ and $x \rightarrow x^{\prime}$ and $x^{\prime} \rightarrow y^{\prime}$ all hold.

2. We have $y \rightarrow_{*} y^{\prime}$ and $x \rightarrow_{*} x^{\prime}, x^{\prime} \rightarrow_{*} y^{\prime}$ imply $x \rightarrow_{*} y$.

3. We seek maximal subsets $\mathbb{E}$ of $S$, called extensions, satisfying the following:

(a) $\mathbb{E}$ is $*$-conflict free, namely for no $x, y \in \mathbb{E}$ do we have $x \rightarrow_{*} y$.

(b) $\mathbb{E}$ can $*$-defend itself, namely if for some $x$ and some $y \in \mathbb{E}, x \rightarrow * y$ then there exists $z \in \mathbb{E}$ such that $z \rightarrow_{*} x$.

Lemma 2.1 Let $\mathbb{E}$ be an extension and let $x \rightarrow_{*} y$ and $x \in \mathbb{E}$ hold then $y \in \mathbb{E}$. 
Proof First we note that $\mathbb{E} \cup\{y\}$ os $*$-conflict free For if some $e \in \mathbb{E}$ either $e \rightarrow_{*} y$ or $y \rightarrow_{*} e$ holds then by (2) above we have that either $\rightarrow_{*} x$ or $x \rightarrow_{*} e$. Then we note that if $z \rightarrow_{*} y$, then $z \rightarrow_{*} x$ (by (2)), and so for some $e \in \mathbb{E}$ we have $e \rightarrow_{*} z$. Thus $\mathbb{E} \cup\{y\}$ is $*$-conflict free and can $*$-defend itself. Since $\mathbb{E}$ is a maximal such set we have $y \in \mathbb{E}$.

Let us now check what extensions we get for Fig. 2. We get $\mathbb{E}=\{u\}$. Certainly $\{u\}$ is $*$-conflict free and can defend itself (it is not attacked). The nodes $\{0, x, y\} *$-attack themselves and so they cannot be added to $\{u\}$. The node $z$ does not $*$-attack itself but it cannot defend itself. It is attacked by $y$ and neither $z$ nor $u$ attack $y$. So $\mathbb{E}=\{u\}$ is maximal.

When the argumentation network is not a tree, we need to use double arrows attacking arrows or nodes attacking arrows. These are known as higher level attacks.

The modal logic papers in this special issue discuss some of the formal properties of reactivity modalities.

The argumentation papers deal with applications of the reactivity idea.

The paper "Reactive Kripke Semantics and Arc Accessibility" introduces the basic ideas of reactivity and discusses some applications. The paper "Reactive Beth Tableaux for Modal Logic" gives a tableaux formulation of some basic reactive modal logics and also shows that the reactive semantics is strictly stronger than ordinary Kripke semantics. The paper "Completeness Theorems for Reactive Modal Logics" addresses the problem of completeness for a basic switch reactive $\mathbf{K}$ modality. The paper "Global View On Reactivity: Switch Graphs and Their Logics" discusses general properties of switch reactive modalities.

There are two argumentation papers in this issue. The first deals with support and the second deals with compliance, both make use of higher level reactivity.

There are two related other papers in this issue. The one on Causal Dynamic Inference is related to reactivity. The theorem proving paper is related only as much as general automated deduction is related to general logic. 